\title{
The Effect of $17 \beta$-Estradiol Administration on Cutaneous Wound Healing in 24-Week Ovariectomized Female Mice
}

\author{
Kanae Mukai, Yukari Nakajima, Tamae Urai, Emi Komatsu, Kana Takata, \\ Yuriko Miyasaka, Nasruddin, Junko Sugama, and Toshio Nakatani \\ Graduate Course of Nursing Science, Division of Health Sciences, Department of Clinical Nursing, \\ Graduate School of Medical, Pharmaceutical and Health Sciences, Kanazawa University, 5-11-80 Kodatsuno, \\ Ishikawa 920-0942, Japan \\ Correspondence should be addressed to Toshio Nakatani; nakatosi@staff.kanazawa-u.ac.jp
}

Received 26 June 2013; Revised 21 November 2013; Accepted 22 November 2013; Published 21 January 2014

Academic Editor: Tullio Florio

Copyright (C) 2014 Kanae Mukai et al. This is an open access article distributed under the Creative Commons Attribution License, which permits unrestricted use, distribution, and reproduction in any medium, provided the original work is properly cited.

\begin{abstract}
Estrogen replacement promotes cutaneous wound healing in 8-10-week young ovariectomized female mice. However, research using aged ovariectomized female mice has not been reported, to the best of our knowledge. Therefore, we investigated the effect of $17 \beta$-estradiol on cutaneous wound healing using 24 -week middle-aged ovariectomized female mice. Twenty-week-old female mice were divided into three groups: medication with $17 \beta$-estradiol after ovariectomy (OVX $+17 \beta$-estradiol), ovariectomy (OVX), and sham (SHAM). After 4 weeks, the mice received two full-thickness wounds. Then, the OVX $+17 \beta$-estradiol group was administered $17 \beta$-estradiol at $0.01 \mathrm{~g} /$ day until healing. The ratio of wound area in the OVX $+17 \beta$-estradiol group was significantly decreased compared with that in the OVX group. The numbers of neutrophils and macrophages in the OVX $+17 \beta$-estradiol group were significantly smaller than those in the OVX group. In addition, the ratio of myofibroblasts in the OVX $+17 \beta$-estradiol group was significantly higher than that in the OVX group. These data suggested that exogenous continuous $17 \beta$-estradiol administration promotes cutaneous wound healing in 24-week OVX female mice by reducing wound area, shortening inflammatory response, and promoting wound contraction. However, it is unclear whether the effect of exogenous estrogen on wound healing outweighs the delay of wound healing due to advanced age.
\end{abstract}

\section{Introduction}

Cutaneous wound healing is a complex, tightly orchestrated response to injury, carefully regulated at temporal and spatial levels [1]. Generally, there are various overlapping phases of the repair process (the immediate response; the inflammatory response; the proliferation, migration, and contraction phase; and the remodeling phase) [1].

With advanced age, this series of events becomes disrupted and healing is delayed. In elderly humans, delayed healing of acute wounds is characterized by the extension of inflammation [2], increased protease activity [3, 4], and delayed re-epithelialization [5]. However, postmenopausal women with systemically reduced estrogen heal poorly [5], whereas exogenous estrogen treatment reverses this delayed cutaneous wound healing by dampening excessive neutrophil recruitment [6], promoting re-epithelialization [5] and increasing collagen deposition [5-7].

Rodent models subjected to ovariectomy (OVX) constitute a cornerstone in elucidation of the effects and detailed biological mechanisms of estrogen. OVX exhibited delayed cutaneous wound healing compared with SHAM $[5,6,8-14]$, whereas exogenous estrogen treatment reversed this delay by decreasing wound area $[5,8,12-14]$, local numbers of neutrophils and macrophages $[8-12,14]$, and wound levels of TNF $\alpha$ [8-12], and by promoting re-epithelialization $[5,9$, 11-14], and collagen deposition [5]. However, these studies evaluating the effects of estrogen used 8-10-week young female mice, and research using aged female mice has not been reported, to the best of our knowledge.

Since the relationship between advanced age and exogenous estrogen treatment in relation to cutaneous wound 
healing remains unknown, we here attempt to verify the effect of estrogen on cutaneous wound healing using an older OVX female mouse model. We investigate the effect of $17 \beta$-estradiol on cutaneous wound healing using 24 -week middle-aged OVX female mice. The aims of the present study are to evaluate how $17 \beta$-estradiol administration promotes cutaneous wound healing in 24-week middle-aged OVX female mice, and which factor has a stronger influence on cutaneous wound healing: estrogen or age.

\section{Materials and Methods}

2.1. Animals. Sixty-three C57BL/6 female mice aged 8 weeks (Sankyo Lab Service Co., Tokyo, Japan) were used in the experiments. They were caged individually in an airconditioned room at $25.0 \pm 2.0^{\circ} \mathrm{C}$ with light from $08: 45$ to 20:45 hours, and water and chow were given freely. The experimental protocol and animal care were in accordance with the Guidelines for the Care and Use of Laboratory Animals of Kanazawa University, Japan (AP-122316).

2.2. Wounding. The mice were bred until 20 weeks. At this time, they were anesthetized by intraperitoneal (IP) injection of pentobarbital sodium $(0.05 \mathrm{mg} / \mathrm{g}$ weight), and the dorsum was shaved. Then, they were subjected to sham surgery (SHAM) or ovariectomy (OVX) according to OECD guidelines [15]. Four weeks later, they were divided into three groups (21 mice/group): SHAM group, OVX group, and OVX $+17 \beta$-estradiol group. Then, under anesthesia with shaving, two circular full-thickness skin wounds $(4 \mathrm{~mm}$ in diameter) including the panniculus carnosus muscle on both sides of the dorsum of the mouse were made with a Kai sterile disposable biopsy punch (Kai Industries Co. Ltd., Gifu, Japan). In SHAM and OVX groups, the wounds were covered with hydrocolloid dressing (Tegaderm; 3M Health Care, Tokyo, Japan) to maintain a moist environment, and then the mouse was wrapped with sticky bandages (Meshpore Tape; Nichiban, Tokyo, Japan). They were changed every day. In the OVX $+17 \beta$-estradiol group, wounds received the same treatment. However, after wound treatment, they were also treated with $0.01 \mathrm{~g}$ of $17 \beta$-estradiol gel (L'estrogel $0.06 \%$; Bayer Yakuhin, Osaka, Japan). It was placed on clean gauze using a 1-mL syringe and applied to the skin on the back avoiding the wounds every day.

2.3. Macroscopic Observation. The day when wounds were made was designated as day 0 , and the process of wound healing was observed from days 0 to 14 after wounding. Wound edges were traced on polypropylene sheets and photographs were taken every day. The traces on the sheets were captured with a scanner onto a personal computer using Adobe Photoshop Elements 7.0 (Adobe System Inc., Tokyo, Japan), and the areas of wounds were calculated using image analysis software Scion Image Beta 4.02 (Scion Corporation, Frederick, Maryland, USA). Wound area is shown as the ratio of wound area every day to the initial wound area on day 0 when the wound was made. Our previous studies reported [16-18] that a full-thickness cutaneous wound on the back of a mouse without any management, such as the application of honey, daidzein, or estrogen, healed with scar formation on about day 14 after wounding and the ratio of wound area reached about 0.1. Therefore, we calculated the number of wounds reaching the 0.1 ratio of wound area per group.

2.4. Plasma $17 \beta$-Estradiol and Uterus Assay. The mice were euthanized by a massive pentobarbital sodium IP injection on day 14. Plasma was prepared from each mouse's blood isolated through cardiac puncture and frozen until the time of assay.Plasma $17 \beta$-estradiol levels were determined by radioimmunoassay (RIA), and since levels less than $10 \mathrm{pg} / \mathrm{mL}$ could not be detected, such levels were recorded as $10 \mathrm{pg} / \mathrm{mL}$. This was outsourced to the manufacturer of this assay (Mitsubishi Chemical Medience Corporation, Tokyo, Japan). The uterus was harvested according to OECD guidelines [15] after blood isolation, its wet weight was measured, and it was fixed in $4 \%$ paraformaldehyde and embedded in paraffin.

2.5. Plasma TNF- $\alpha$. The mice were euthanized by a massive pentobarbital sodium IP injection on days 3 and 7. Plasma was prepared from each mouse's blood isolated through cardiac puncture and frozen until the time of assay. Plasma TNF$\alpha$ levels were determined by ELISA (R\&D Systems, Tokyo, Japan) according to the manufacturer's guidelines.

\subsection{Histological Procedure and Immunohistological Staining.} The mice were euthanized by a massive pentobarbital sodium IP injection on days $3,7,11$, and 14 after wounding. The wound and the surrounding intact skin were harvested and each sample of wound and surrounding intact skin was bisected at the wound center. One-half of each wound was stapled onto polypropylene sheets to prevent overcontraction of the sample and fixed in $4 \%$ paraformaldehyde for 12 hours. These samples were dehydrated in an alcohol series, cleaned in xylene, and embedded in paraffin to prepare $5 \mu \mathrm{m}$ serial paraffin sections. The remainder of each wound was embedded in tissue-Tek OCT (Sakura Finetek, Japan) before fixing to prepare $5-\mu \mathrm{m}$ serial ice sections. At least 6 serial sections near the center of the wound were obtained from one wound and stained according to the following methods. Paraffin sections of 5- $\mu \mathrm{m}$ thickness were stained with hematoxylin and eosin (H\&E) or subjected to Azan staining and immunohistologically stained with antineutrophil antibody at a concentration of 1:100 (Abcam Japan, Tokyo, Japan) for detecting neutrophils, anti-Mac-3 antibody at a concentration of $1: 100$ (BD Pharmingen, Tokyo, Japan) for detecting macrophages, and cryosections of 5$\mu \mathrm{m}$ thickness were immunohistologically stained with anti$\alpha$-smooth muscle actin ( $\alpha$-SMA) antibody at a concentration of 1:500 (Abcam Japan, Tokyo, Japan) for detecting myofibroblasts. Negative control slides were obtained by omitting each primary antibody.

2.7. Microscopic Observations. Images were imported onto a computer using a digital microscopic camera (DP2-BSW Olympus, Japan). Measurements for the proportions lacking 

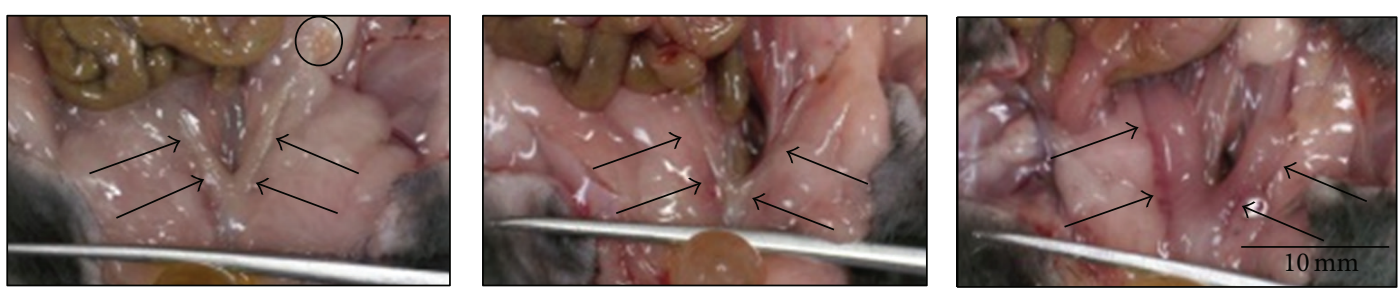

(a)

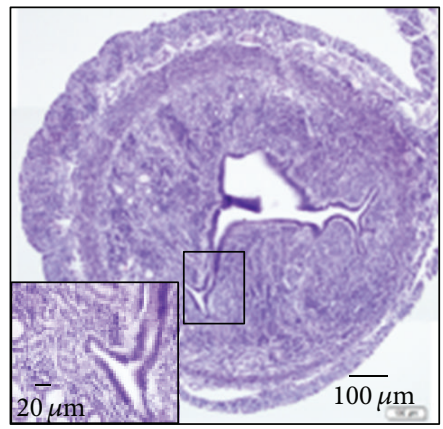

SHAM

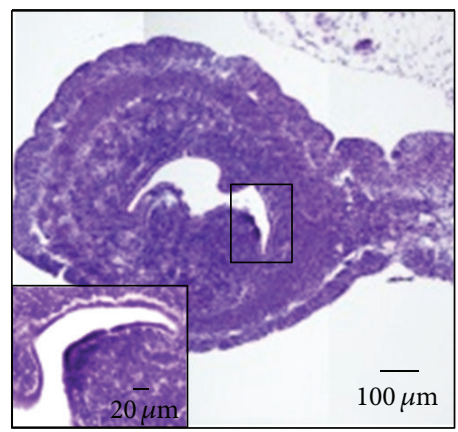

OVX

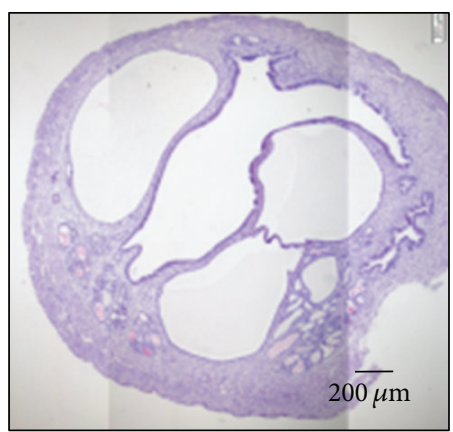

OVX $+17 \beta$-estradiol

(b)

FIgURE 1: Uterus. (a) Macroscopic photos. A circle indicates the ovary and arrows indicate the uterus. In the SHAM group, the uterus was thin but soft. In the OVX group, it looked thinner than that in the SHAM group and was scleroid and atrophic. On the other hand, in the OVX $+17 \beta$-estradiol group, it was hypertrophic and fresh. (b) Hematoxylin and eosin staining. In the SHAM group, histological features included anestrus, with the epithelium invaginated to endometrium. In the OVX group, invagination of the epithelium had almost disappeared. Otherwise, in the OVX $+17 \beta$-estradiol group, proestrus was shown and the uterine cavity was extended.

re-epithelialization were performed using DP2-BSW Olympus software: the distance between both wound edges and the distance between the tips of elongated new epithelium were measured, and then the latter was divided by the former (no re-epithelialization length/wound length). Measurements for collagen deposition colored blue (collagen pixels/total wound pixels) and for myofibroblasts colored brown (myofibroblast pixels/total wound pixels) were performed using Adobe Photoshop Elements 7.0 as follows: the wound area was first selected; one wound edge, the surface of the wound, the other wound edge, and the bottom of the wound, which is the position of the panniculus carnosus, were surrounded, and then the number of pixels in the surrounded area $(=$ wound area) was calculated. Next, the collagen deposition colored blue or myofibroblasts colored brown were selected and the number of pixels of the blue or brown area (the area of collagen deposition or the area of myofibroblasts) was calculated; finally, the number of pixels of the area of collagen deposition or the number of pixels of the area of myofibroblasts was divided by the number of pixels of the wound area. To analyze the numbers of neutrophils and macrophages in the granulation tissue, each positive cell was counted by observation through a light microscope using a $\times 40$ objective at three sites of granulation tissue: two sites near the two wound edges and the center of the granulation tissue. Areas of these three sites were calculated on the monitor of the DP2-BSW and the total number of neutrophils or macrophages at the three sites was divided by the whole area of these three sites.
2.8. Statistical Analysis. Data are expressed as mean $\pm \mathrm{SD}$ and analyzed using JMP 8.0.1 (SAS, USA). Fisher's exact probability test, ANOVA, and Student's $t$-test or TukeyKramer multiple comparison test was performed. Differences were considered significant at $P<0.05$.

\section{Results}

3.1. Uterine Weight and Plasma $17 \beta$-Estradiol Levels. We confirmed that the ovaries had been removed successfully in the OVX and OVX $+17 \beta$-estradiol groups. The body weight on day 14 after wounding was $25.08 \pm 0.90 \mathrm{~g}$ in the SHAM group, $25.45 \pm 0.38 \mathrm{~g}$ in the OVX group, and $25.33 \pm 2.67 \mathrm{~g}$ in the OVX $+17 \beta$-estradiol group. The macroscopic and histological features of the uterus are shown in Figures 1(a) and $1(\mathrm{~b})$. The uterine weight in the OVX $+17 \beta$-estradiol group was significantly greater than the uterine weights of SHAM and OVX groups on day $14(P<0.01)$. There were no significant differences between SHAM and OVX groups on day 14 (Table 1). The plasma $17 \beta$-estradiol level in the OVX + $17 \beta$-estradiol group was significantly greater than those levels of SHAM and OVX groups on day $14(P<0.01)$. There were no significant differences between SHAM and OVX groups on day 14 (Table 1), although in the OVX group, 4 out of 6 mice had plasma $17 \beta$-estradiol levels under $10 \mathrm{pg} / \mathrm{mL}$ and, in the SHAM group, 1 out of 6 mice did. Estrus and proestrus were not observed in the SHAM group when we observed smears of the SHAM mice before euthanizing them. 
TABLE 1: Plasma $17 \beta$-estradiol levels and uterine weight.

\begin{tabular}{lccr}
\hline & SHAM & OVX & OVX + 17 $\beta$-estradiol \\
\hline $17 \beta$-estradiol $(\mathrm{pg} / \mathrm{mL})$ & $11.3 \pm 1.0^{* *}$ & $10.8 \pm 1.8^{* * *}$ & $27.7 \pm 7.7$ \\
Uterine weight $(\mathrm{mg})$ & $35.0 \pm 9.0^{* *}$ & $34.0 \pm 16.0^{* *}$ & $122.0 \pm 21.0$ \\
\hline
\end{tabular}

Values are expressed as mean $\pm \mathrm{SD}, n=6$ for each group, ANOVA, Tukey-Kramer. ${ }^{* *} P<0.01$ with respect to the OVX $+17 \beta$-estradiol group.

3.2. Wound Area. In the SHAM group, wound areas increased for 2 days and then decreased rapidly until day 8 , after which they decreased slowly until day $14(0.15 \pm 0.08$, ratio of wound area to initial wound area on day 14). The ratio of wound area to initial wound area of 5 out of 12 wounds reached a ratio of 0.1 to the initial wound area on day 14 . In the OVX group, wound areas increased for 2 days and then decreased rapidly until day 9 , after which they decreased slowly until day $14(0.24 \pm 0.15)$. The ratio of wound area to initial wound area of none out of 12 wounds reached a ratio of 0.1 on day 14 . On the other hand, in the OVX $+17 \beta$ estradiol group, wound area increased only for 1 day and then decreased rapidly until day 11 , after which it decreased slowly until day $14(0.08 \pm 0.03)$. The ratio of wound area to initial wound area of 9 out of 12 wounds reached a ratio of 0.1 on day 14 . The ratio of wound area in the OVX $+17 \beta$-estradiol group was significantly smaller than that of the OVX group on days 4 and $11-14(P=0.0397,0.0203,0.0065,0.0024$, and 0.0014 , resp.). There were no significant differences of the ratio of wound area between OVX $+17 \beta$-estradiol and SHAM groups, or between SHAM and OVX groups on days $0-14$. However, the mean ratio of wound area in the OVX group was larger throughout the whole period, except for the first day (Figure 2(b)). The number of wounds reaching a ratio of wound area of 0.1 on day 14 after wounding was significantly different between SHAM and OVX groups and between OVX and OVX $+17 \beta$-estradiol groups $(P=0.0373$ and 0.0003 , resp.), but there were no significant differences between SHAM and OVX $+17 \beta$-estradiol groups.

3.3. Neutrophils, Macrophages and Plasma TNF- $\alpha$ Levels. The number of neutrophils in the OVX $+17 \beta$-estradiol group was significantly smaller than that in the OVX group on day 3 $(P=0.0326)$. There were no significant differences between OVX $+17 \beta$-estradiol and SHAM groups or between SHAM and OVX groups on day 3 , and there were no significant differences among the three groups on day 7 (Figure 3(a)). The numbers of neutrophils significantly decreased from days 3 to 7 in the OVX and OVX $+17 \beta$-estradiol groups $(P=$ 0.0137 and 0.0145 , resp.).

The number of macrophages in the OVX $+17 \beta$-estradiol group was significantly smaller than that in the OVX group on day $3(P=0.0012)$. There were no significant differences between OVX $+17 \beta$-estradiol and SHAM groups, or between SHAM and OVX groups on day 3 , and there were no significant differences among the three groups on day 7 (Figure 3(c)). The number of macrophages decreased in the OVX group from days 3 to 7 but remained unchanged in the SHAM and OVX $+17 \beta$-estradiol groups.

There were no significant differences of plasma TNF- $\alpha$ levels among the three groups on days 3 and 7 (Figure 3(e)).

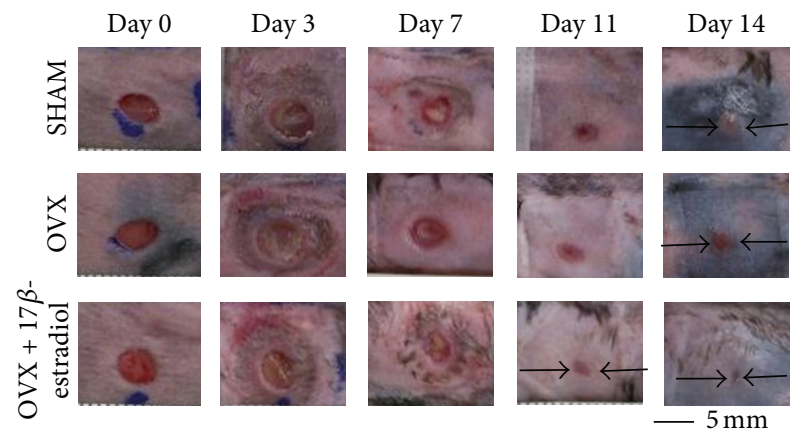

(a)

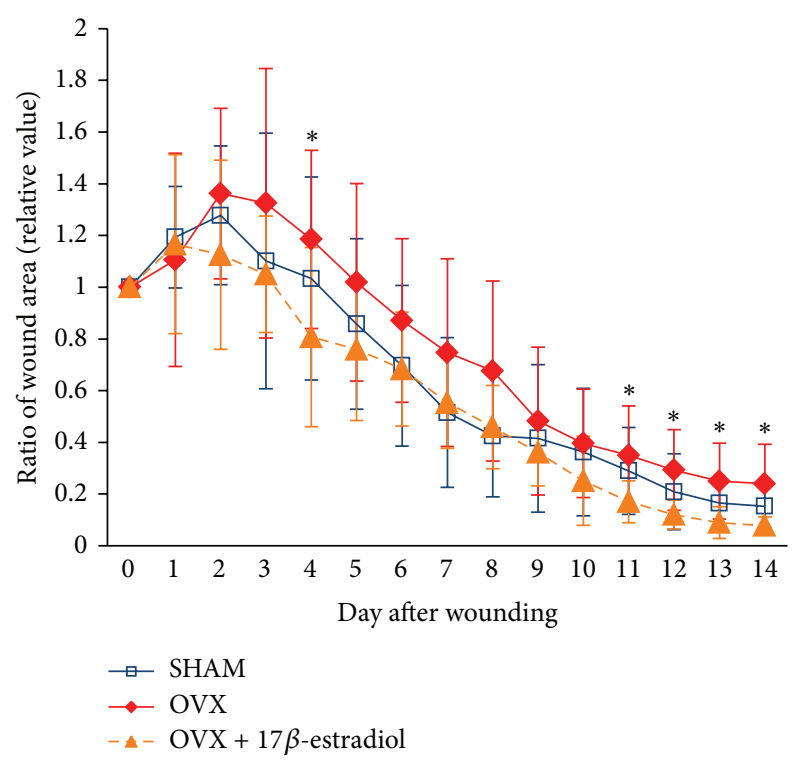

(b)

FIGURE 2: Macroscopic wound healing. (a) Four mm diameter wounds were produced and healing was recorded by photography. Bar $5 \mathrm{~mm}$. (b) The ratios of wound areas to initial area on day 0 are shown on line graphs for each day. There were significant differences between the OVX $+17 \beta$-estradiol and OVX groups on days 4 and 11-14. However, there were no significant differences between the SHAM and OVX groups or between the SHAM and OVX $+17 \beta$ estradiol groups. Values are expressed as mean $\pm \mathrm{SD}, n=12$, for each group, ANOVA, Tukey-Kramer, ${ }^{*} P<0.05$ : OVX versus OVX $+17 \beta$-estradiol.

Plasma TNF- $\alpha$ levels in the SHAM and OVX $+17 \beta$-estradiol groups decreased from days 3 to 7 , with a significant decrease in the OVX $+17 \beta$-estradiol group $(P=0.0233)$, but it remained unchanged in the OVX group.

3.4. Re-Epithelialization, Collagen Deposition, and Wound Contraction. On day 3, new epithelium appeared from the 


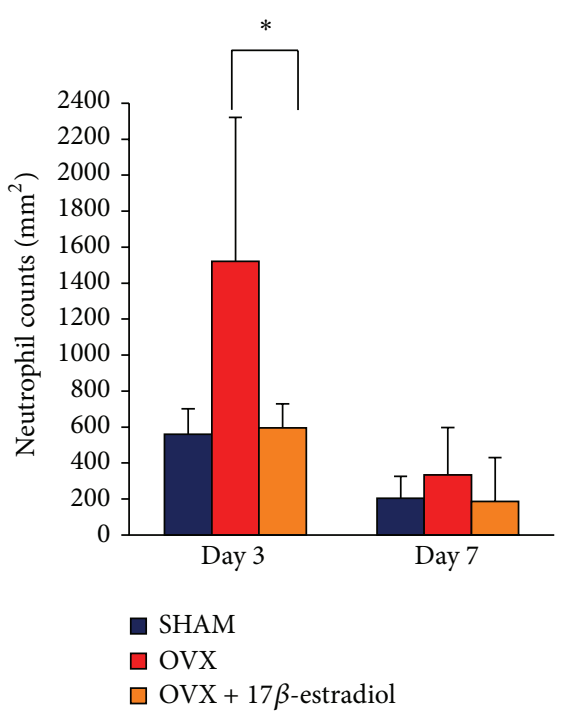

(a)

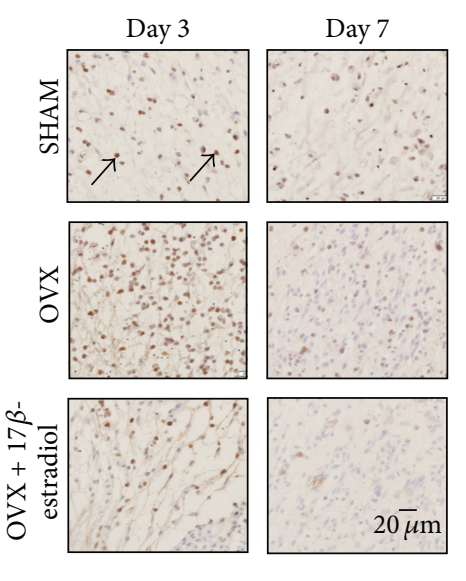

(b)

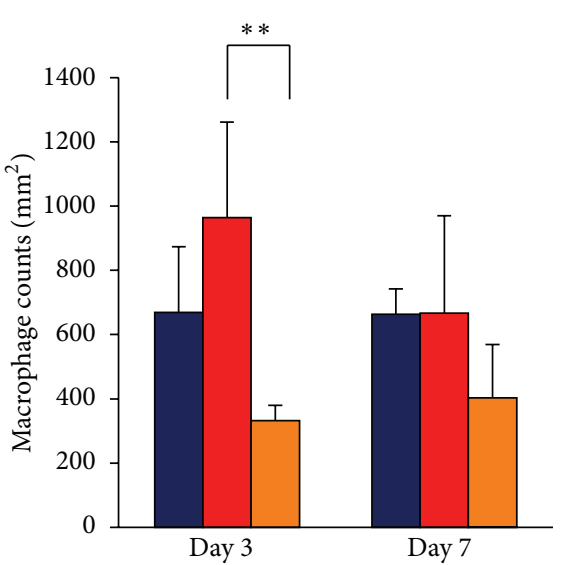

— SHAM

$\square$ OVX

$\square$ OVX $+17 \beta$-estradiol

(c)
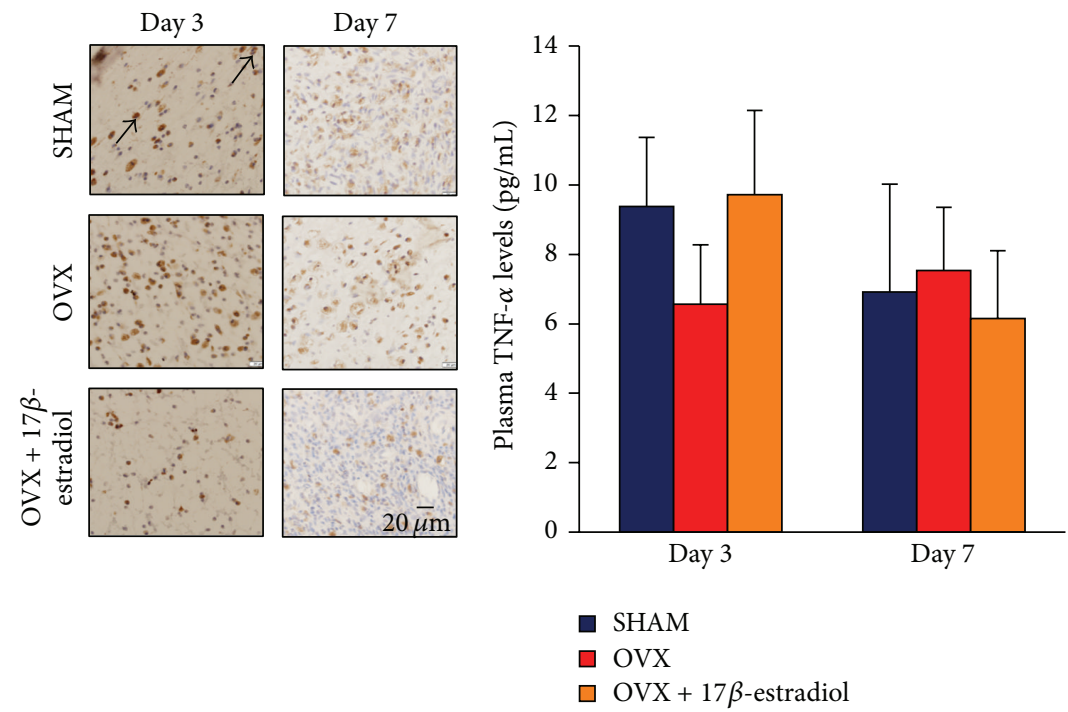

(d)

(e)

FIGURE 3: Inflammatory cells and plasma TNF- $\alpha$ levels. (a) The number of neutrophils per $\mathrm{mm}^{2}$. The number of neutrophils in the OVX $+17 \beta$-estradiol group was significantly smaller than that in the OVX group on day 3. (b) Neutrophils (arrows) stained with antineutrophil antibody were observed in wound tissue on days 3 and 7. Bar $20 \mu \mathrm{m}$. (c) The number of macrophages per mm ${ }^{2}$. The number of macrophages in the OVX $+17 \beta$-estradiol group was significantly smaller than that in the OVX group on day 3. (d) Macrophages (arrows) stained with anti-Mac-3 antibody were observed in wound tissue on days 3 and 7. Bar $20 \mu \mathrm{m}$. (e) Systemic TNF- $\alpha$ levels, determined by ELISA on serum samples. There were no significant differences between the three groups on days 3 and 7 . Values are expressed as mean $\pm \mathrm{SD}, n=4-5$ for each group, ANOVA, $t$-test, or Tukey-Kramer; ${ }^{*} P<0.05,{ }^{* *} P<0.01$ : OVX versus OVX $+17 \beta$-estradiol.

wound edge. It gradually covered the wound surface along with wound healing. By day 11, new epithelium completely covered the wound surface in the OVX $+17 \beta$-estradiol group, but 1 out of 5 wounds was not completely covered in the SHAM and OVX groups. By day 14, the surfaces of all wounds in the three groups were covered with new epithelium. However, there were no significant differences among the three groups on days 3-14 (Figure 4(a)).
Collagen deposition became thick and had a high density with the progression of wound healing in the three groups. There were no significant differences among the three groups on days $7-14$, but that in the OVX $+17 \beta$-estradiol group tended to be larger than that in the OVX group on days 7 and $11(P=0.0648$ and 0.0799 , resp. $)$ (Figure $4(\mathrm{c}))$.

A few myofibroblasts were observed at the wound site in the three groups on day 7 . By day 11 , many myofibroblasts 


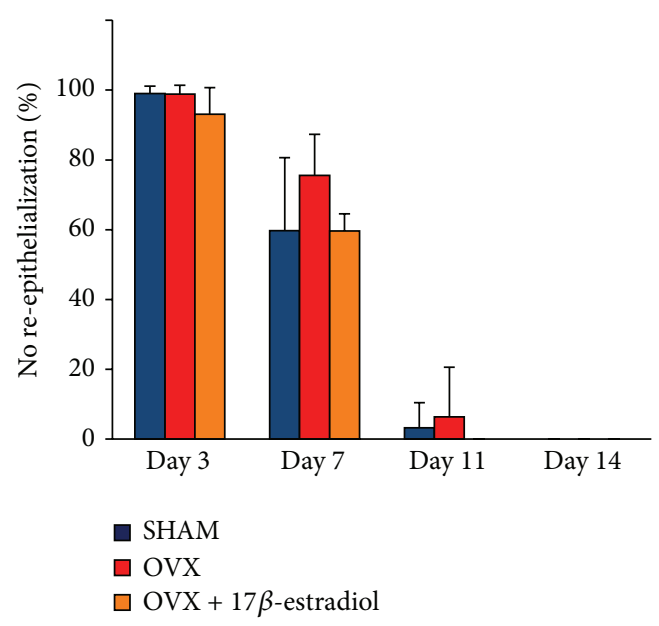

(a)

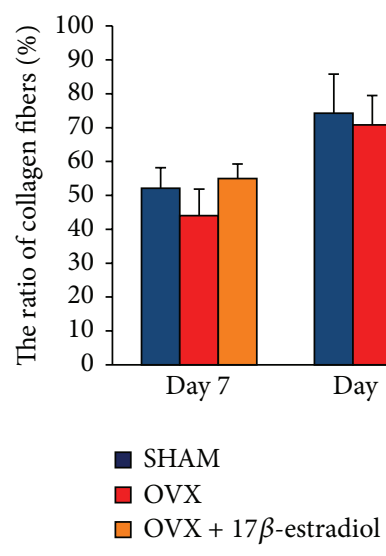

(c)

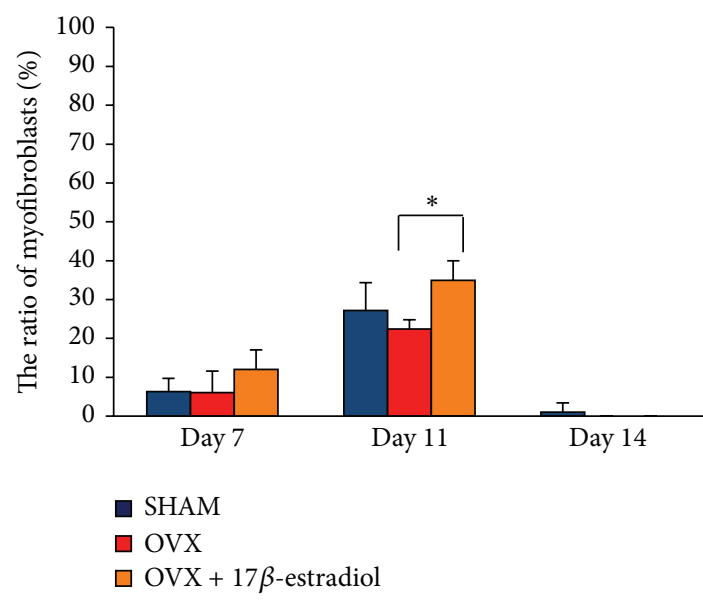

(e)
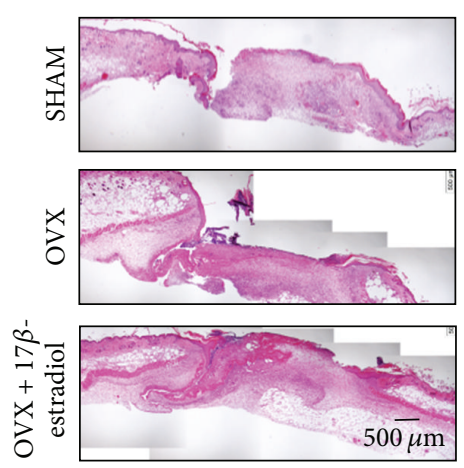

(b)

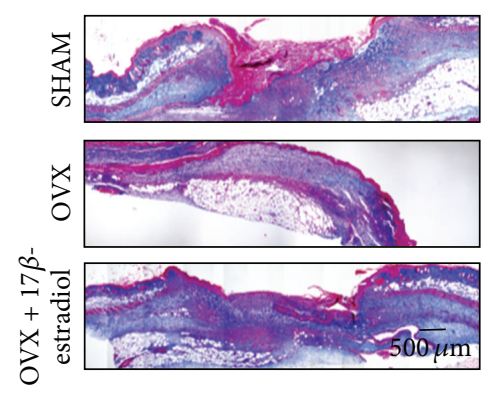

(d)
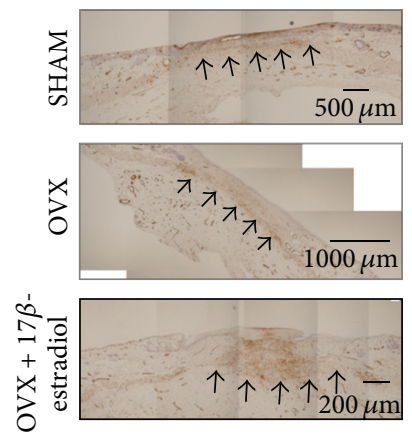

(f)

FIGURE 4: Re-epithelialization, collagen deposition, and wound contraction. (a) Proportion of no re-epithelialization. There were no significant differences between the three groups on days 3-14. (b) Hematoxylin and eosin sections on day 7. Bar 500 $\mu \mathrm{m}$. (c) The ratio of collagen fibers. There were no significant differences between the three groups, although that in the OVX $+17 \beta$-estradiol group tended to be increased compared with that in the OVX group on days 7 and 11. (d) Azan-stained sections on day 7. Bar 500 $\mu \mathrm{m}$. (e) The ratio of myofibroblasts. The ratio of myofibroblasts in the OVX $+17 \beta$-estradiol group increased significantly compared with that in the OVX group on day 11. However, there were no significant differences between OVX $+17 \beta$-estradiol and SHAM groups or SHAM and OVX groups. (f) Myofibroblasts stained with anti- $\alpha$-SMA antibody were observed in granulation tissue on day 11. Bars $500 \mu \mathrm{m}$ (SHAM), $1000 \mu \mathrm{m}$ (OVX), and $200 \mu \mathrm{m}(\mathrm{OVX}+17 \beta$-estradiol). Note a bridge-like structure (arrows) formed by the myofibroblasts. Values are expressed as mean $\pm \mathrm{SD}$, $n=4-5$ for each group, ANOVA, Tukey-Kramer; ${ }^{*} P<0.05$ : OVX versus OVX $+17 \beta$-estradiol. 
were observed in the granulation tissue in the three groups, building bridge-like structures. They had almost completely disappeared by day 14 , with no detection in the three groups. The ratio of myofibroblasts in the OVX $+17 \beta$-estradiol group was significantly larger than that in the OVX group on day 11 $(P=0.0426)$. However, there were no significant differences between OVX $+17 \beta$-estradiol and SHAM groups or between SHAM and OVX groups on days 7-14 (Figure 4(e)).

\section{Discussion}

In the present study, the ratio of wound area in the OVX + $17 \beta$-estradiol group was significantly smaller than that in the OVX group on days 4 and 11-14 after wounding. This shows that exogenous, continuous $17 \beta$-estradiol administration is effective for decreasing wound area in 20-week OVX female mice. This agrees with previous research that cutaneous wound healing in OVX mice is promoted by exogenous estrogen treatment via a decrease of wound area $[5,8,12-$ 14]. On the other hand, in the present study, the ratio of wound area was not significantly different between SHAM and OVX $+17 \beta$-estradiol groups. The same result can be assumed from graphs in previous research papers, although they do not clearly show that cutaneous wound healing in SHAM mice was not delayed compared with that in OVX mice administered $17 \beta$-estradiol at the age of 8-10 weeks [911]. Moreover, in the present study, the ratio of wound area was also not significantly different between SHAM and OVX groups. This contradicts previous research that cutaneous wound healing in OVX mice was delayed compared with that in SHAM mice [5, 6, 8-14]. However, the number of wounds reaching a ratio of wound area of 0.1 on day 14 after wounding in the present study in the SHAM group was significantly larger than that in the OVX group. This shows that cutaneous wound healing in SHAM mice is promoted compared with that in OVX mice by physiological estrogen. From these findings, it is assumed that cutaneous wound healing in OVX mice is the most delayed, and cutaneous wound healing in OVX mice with exogenous, continuous $17 \beta$-estradiol administration is the most promoted, while cutaneous wound healing in SHAM mice is intermediate between these two groups. These findings indicate that exogenous, continuous $17 \beta$-estradiol administration is more effective for cutaneous wound healing than the natural physiological $17 \beta$-estradiol that fluctuates with estrus [19].

These differences in cutaneous wound healing among OVX, SHAM, and OVX $+17 \beta$-estradiol groups in the present study cannot necessarily be interpreted by the present results: the ratio of re-epithelialization, the numbers of neutrophils and macrophages, the level of TNF- $\alpha$, and the ratios of myofibroblasts and collagen deposition. Previous researchers reported that the physiological level of $17 \beta$-estradiol and exogenous, continuous $17 \beta$-estradiol administration after OVX promoted cutaneous wound healing by promoting reepithelialization $[5,9,11-14]$, decreasing local numbers of neutrophils and macrophages $[8-12,14]$ as well as wound level of TNF $\alpha$ [8-12], and promoting collagen deposition [5]. In addition, Emmerson et al. reported that smooth muscle action (SMA) was reduced in day 7 wound tissue from OVX mice compared with that of wild-type mice, which have the physiological level of $17 \beta$-estradiol [13]. On the other hand, in the present study, although there were significant differences only between OVX and OVX $+17 \beta$-estradiol groups-the numbers of neutrophils and macrophages in the OVX + $17 \beta$-estradiol group were significantly smaller than those in the OVX group on day 3 , and the ratio of myofibroblasts in the OVX $+17 \beta$-estradiol group increased significantly more than that of the OVX group on day 11-there were no significant differences in the ratios of collagen deposition and re-epithelialization or the level of TNF- $\alpha$ between OVX and OVX $+17 \beta$-estradiol groups. However, there were no significant differences of these factors between OVX and SHAM groups. These findings may indicate that exogenous, continuous $17 \beta$-estradiol administration is more effective than the natural physiological estrogen that fluctuates over the course of an estrous cycle, as we have discussed for the wound area.

However, from these findings, the following question arises. Why were there no significant differences between OVX and SHAM mice? Previous research evaluated the effects of estrogen on cutaneous wound healing using 8-10week young female mice in comparison among OVX, SHAM, and OVX with exogenous $17 \beta$-estradiol administration. In the present study, in contrast to previous research, we used aged 24-week mice. Mills et al. demonstrated that cutaneous wound healing in old male mice was delayed by extending the wound area, compared with that in young male mice [20]. Hardman and Ashcroft reported, from a microarray study, that $78 \%$ of differentially expressed genes were estrogenregulated, while only $3 \%$ were age-associated [21]. The results in the present study suggest that, although exogenous, continuous $17 \beta$-estradiol administration influences cutaneous wound healing, the influence of age cannot be disregarded since the effect of the SHAM group with physiological estrogen on cutaneous wound healing seems to be intermediate between the effects of the OVX and OVX $+17 \beta$-estradiol groups. Therefore, we will conduct further research using an older mouse model and attempt to evaluate the influence of $17 \beta$-estradiol and advanced age on cutaneous wound healing in the near future.

\section{Conclusions}

We clarified that exogenous, continuous $17 \beta$-estradiol administration has beneficial effects on wound area, inflammatory cells, and myofibroblasts during cutaneous wound healing. Therefore, our research suggests that exogenous, continuous $17 \beta$-estradiol administration also promotes cutaneous wound healing in 24-week OVX female mice by reducing wound area, shortening inflammatory response, and promoting wound contraction. However, it is unclear whether the effect of exogenous, continuous $17 \beta$-estradiol administration on wound healing outweighs the delay of wound healing due to advanced age. 


\section{Conflict of Interests}

The authors declare that there is no conflict of interests regarding the publication of this paper.

\section{Acknowledgment}

Part of this work was supported by Grants-in-Aid for Scientific Research, Japan (nos. 22592363 and 25293430).

\section{References}

[1] T. J. Shaw and P. Martin, "Wound repair at a glance," Journal of Cell Science, vol. 122, part 18, pp. 3209-3213, 2009.

[2] G. S. Ashcroft, M. A. Horan, and M. W. J. Ferguson, "Aging alters the inflammatory and endothelial cell adhesion molecule profiles during human cutaneous wound healing," Laboratory Investigation, vol. 78, no. 1, pp. 47-58, 1998.

[3] S. Herrick, G. Ashcroft, G. Ireland, M. Horan, C. McCollum, and M. Ferguson, "Up-regulation of elastase in acute wounds of healthy aged humans and chronic venous leg ulcers are associated with matrix degradation," Laboratory Investigation, vol. 77, no. 3, pp. 281-288, 1997.

[4] G. S. Ashcroft, M. A. Horan, S. E. Herrick, R. W. Tarnuzzer, G. S. Schultz, and M. W. J. Ferguson, "Age-related differences in the temporal and spatial regulation of matrix metalloproteinases (MMPs) in normal skin and acute cutaneous wounds of healthy humans," Cell and Tissue Research, vol. 290, no. 3, pp. 581-591, 1997.

[5] G. S. Ashcroft, J. Dodsworth, E. van Boxtel et al., "Estrogen accelerates cutaneous wound healing associated with an increase in TGF- $\beta 1$ levels," Nature Medicine, vol. 3, no. 11, pp. 1209-1215, 1997.

[6] G. S. Ashcroft, T. Greenwell-Wild, M. A. Horan, S. M. Wahl, and M. W. J. Ferguson, "Topical estrogen accelerates cutaneous wound healing in aged humans associated with an altered inflammatory response," The American Journal of Pathology, vol. 155, no. 4, pp. 1137-1146, 1999.

[7] E. D. Son, J. Y. Lee, S. Lee et al., "Topical application of $17 \beta$-estradiol increases extracellular matrix protein synthesis by stimulating TGF- $\beta$ signaling in aged human skin in vivo," Journal of Investigative Dermatology, vol. 124, no. 6, pp. 11491161, 2005.

[8] G. S. Ashcroft, S. J. Mills, K. Lei et al., "Estrogen modulates cutaneous wound healing by downregulating macrophage migration inhibitory factor," Journal of Clinical Investigation, vol. 111, no. 9, pp. 1309-1318, 2003.

[9] E. Emmerson, L. Campbell, G. S. Ashcroft, and M. J. Hardman, "The phytoestrogen genistein promotes wound healing by multiple independent mechanisms," Molecular and Cellular Endocrinology, vol. 321, no. 2, pp. 184-193, 2010.

[10] M. J. Hardman, E. Emmerson, L. Campbell, and G. S. Ashcroft, "Selective estrogen receptor modulators accelerate cutaneous wound healing in ovariectomized female mice," Endocrinology, vol. 149, no. 2, pp. 551-557, 2008.

[11] C. E. Routley and G. S. Ashcroft, "Effect of estrogen and progesterone on macrophage activation during wound healing," Wound Repair and Regeneration, vol. 17, no. 1, pp. 42-50, 2009.

[12] M. Brufani, F. Ceccacci, L. Filocamo et al., "Novel locally active estrogens accelerate cutaneous wound healing. A preliminary study," Molecular Pharmaceutics, vol. 6, no. 2, pp. 543-556, 2009.
[13] E. Emmerson, L. Campbell, G. S. Ashcroft, and M. J. Hardman, "Unique and synergistic roles for $17 \beta$-estradiol and macrophage migration inhibitory factor during cutaneous wound closure are cell type specific," Endocrinology, vol. 150, no. 6, pp. 2749-2757, 2009.

[14] L. Campbell, E. Emmerson, F. Davies et al., "Estrogen promotes cutaneous wound healing via estrogen receptor $\beta$ independent of its antiinflammatory activities," Journal of Experimental Medicine, vol. 207, no. 9, pp. 1825-1833, 2010.

[15] OECD, "Uterotrophic bioassay in rodents: a short-term screening test for oestrogenic properties," in OECD Guideline for the Testing of Chemicals, no. 440, 2007.

[16] Haryanto, T. Urai, K. Mukai, Suriadi, J. Sugama, and T. Nakatani, "Effectiveness of Indonesian honey on the acceleration of cutaneous wound healing: an experimental study in mice," WOUNDS, vol. 24, no. 4, pp. 110-119, 2012.

[17] K. Mukai, M. Nagasawa, E. Nakamura et al., "The effect of isoflavone-daidzein oral medication on cutaneous wound healing in female ovariectomized mice," Structure and Function, vol. 10, no. 2, pp. 94-100, 2012.

[18] Y. Nakajima, Y. Eno, M. Hirata et al., "Is estrogen effective for full-thickness cutaneous wound healing in young male mice?" WOUNDS, vol. 25, no. 10, pp. 278-286, 2013.

[19] J. F. Nelson, L. S. Felicio, H. H. Osterburg, and C. E. Finch, "Altered profiles of estradiol and progesterone associated with prolonged estrous cycles and persistent vaginal cornification in aging C57BL/6J mice," Biology of Reproduction, vol. 24, no. 4, pp. 784-794, 1981.

[20] S. J. Mills, J. J. Ashworth, S. C. Gilliver, M. J. Hardman, and G. S. Ashcroft, "The sex steroid precursor DHEA accelerates cutaneous wound healing via the estrogen receptors," Journal of Investigative Dermatology, vol. 125, no. 5, pp. 1053-1062, 2005.

[21] M. J. Hardman and G. S. Ashcroft, "Estrogen, not intrinsic aging, is the major regulator of delayed human wound healing in the elderly," Genome Biology, vol. 9, no. 5, article R80, 2008. 


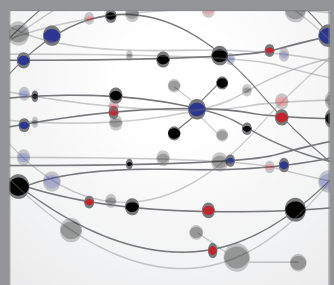

The Scientific World Journal
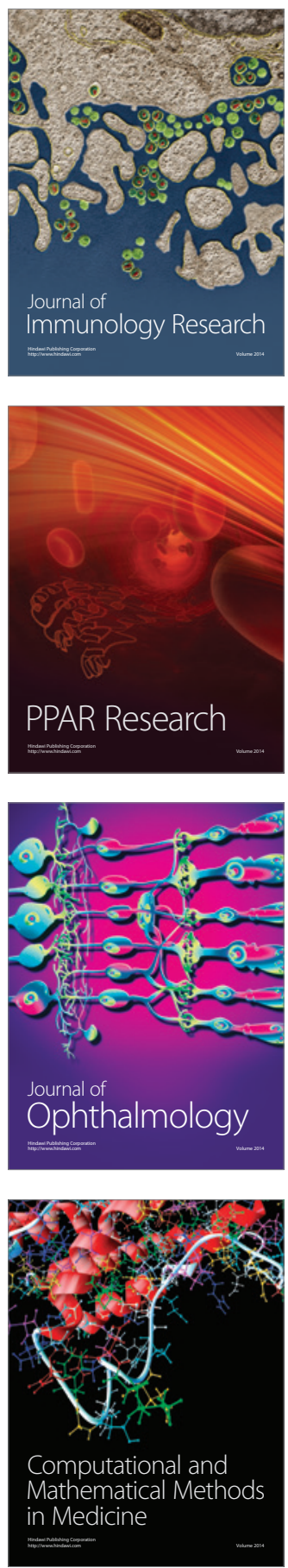

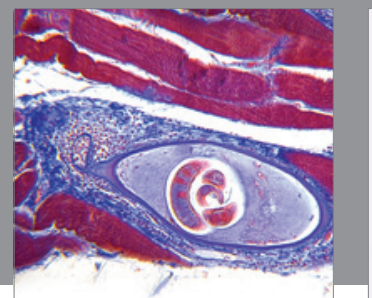

Gastroenterology

Research and Practice
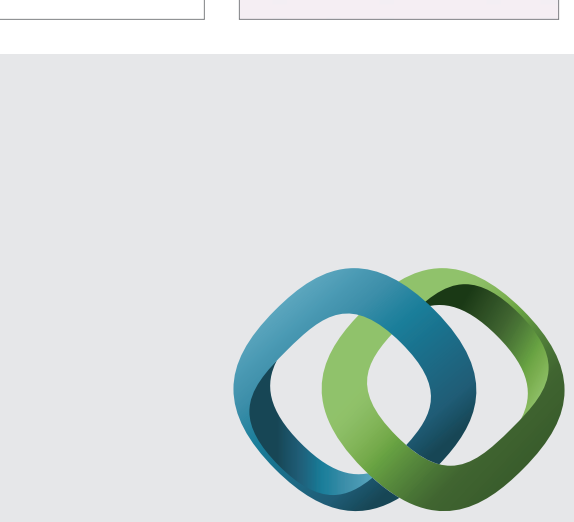

\section{Hindawi}

Submit your manuscripts at

http://www.hindawi.com
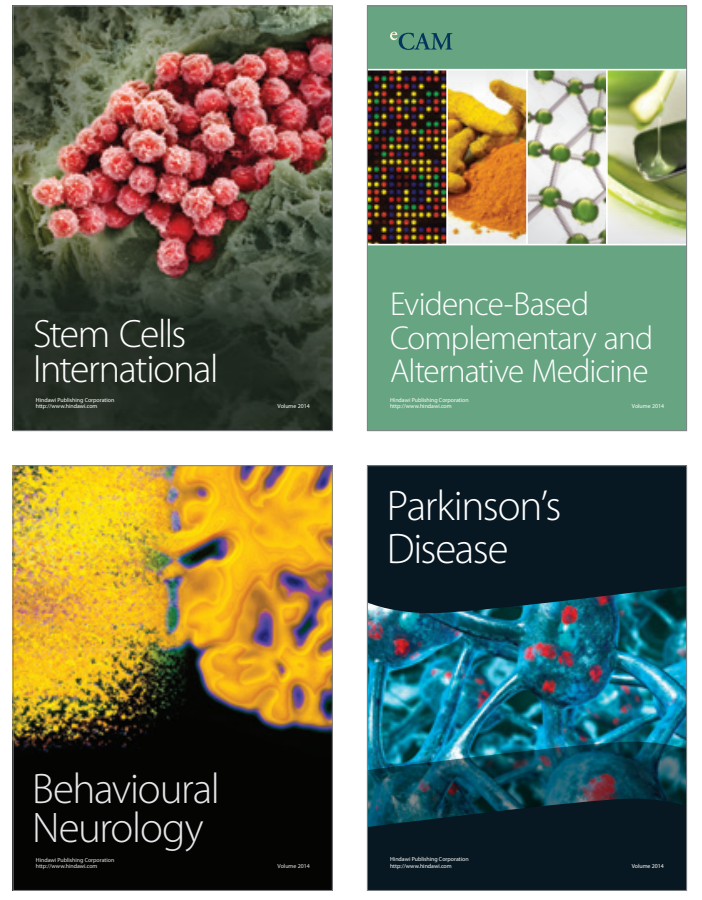
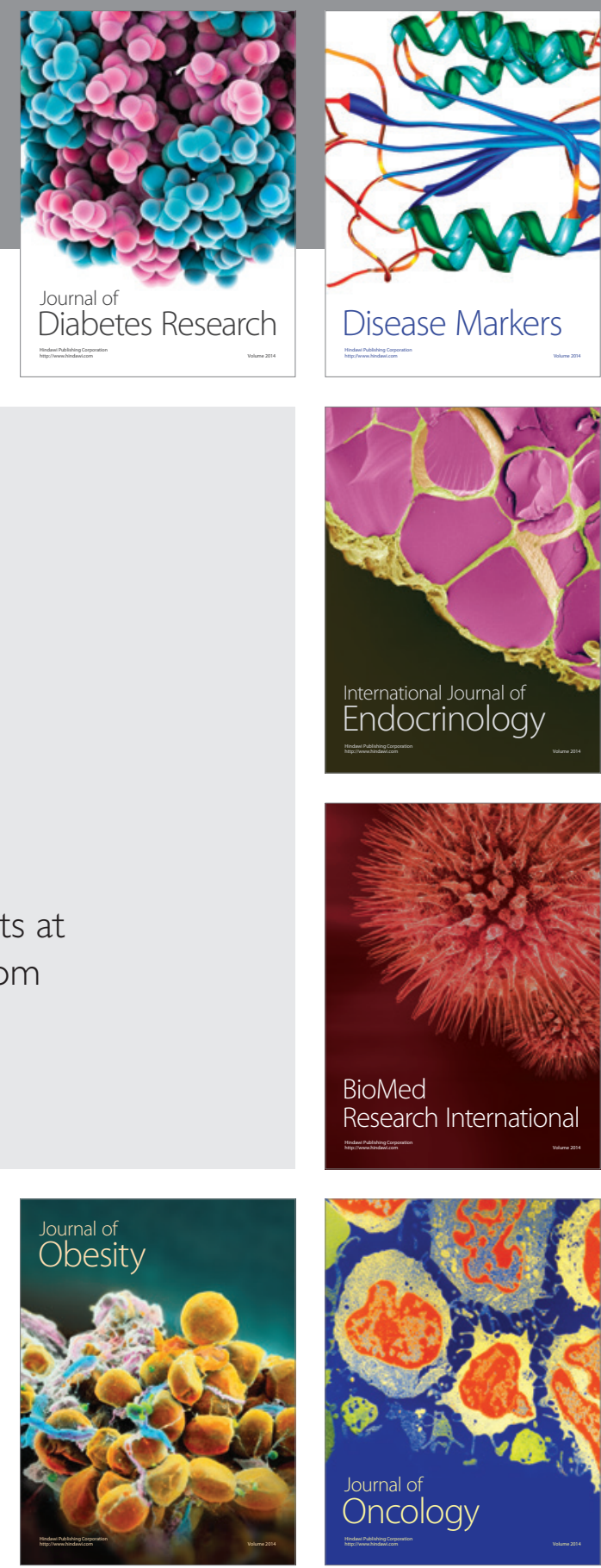

Disease Markers
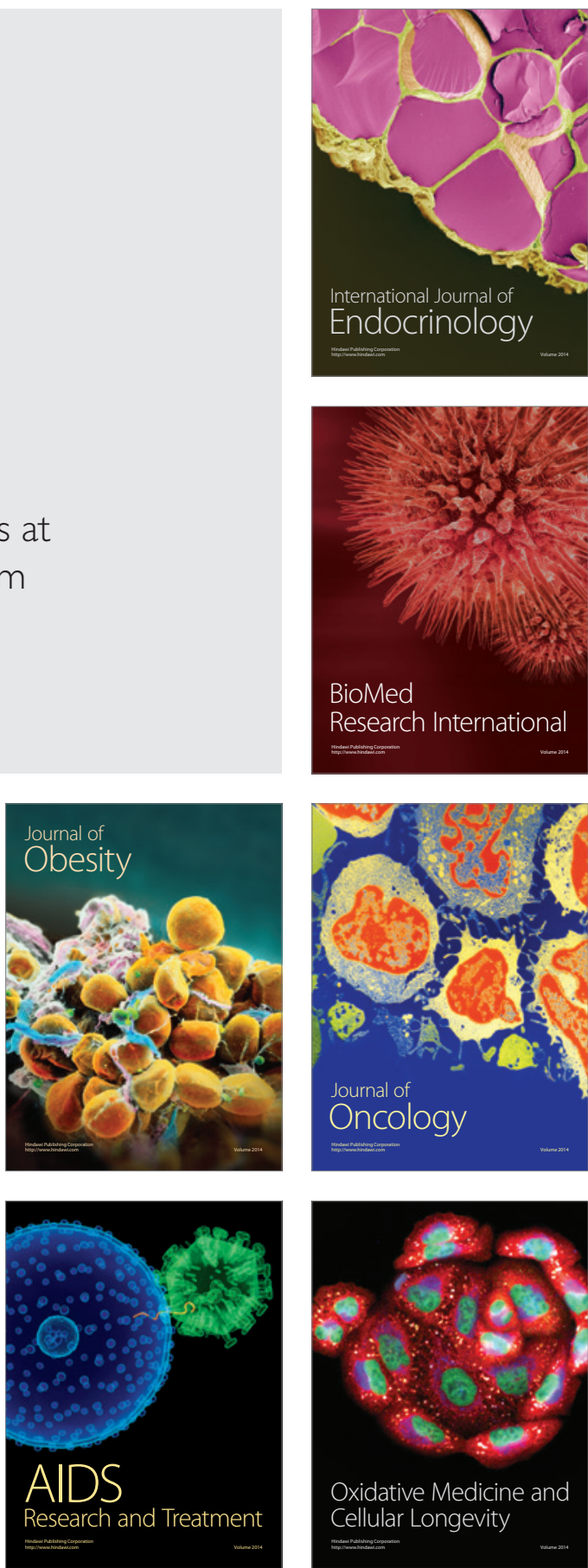